\title{
Enfermedad de Morquio (mucopolisacaridosis IV-A): aspectos clínicos, diagnósticos y nuevo tratamiento con terapia de reemplazo enzimático
}

\author{
Morquio disease (Mucopolysaccharidosis type IV-A): clinical \\ aspects, diagnosis and new treatment with enzyme replacement \\ therapy
}

\author{
Dr. Juan Politei ${ }^{a}$, Dra. Andrea B. Schenone ${ }^{a}$, Dr. Norberto Guelbert ${ }^{b}$, \\ Dr. Alejandro Fainboim ${ }^{c}$ y Dra. Marina Szlago ${ }^{a}$
}

\begin{abstract}
RESUMEN
LamucopolisacaridosistipoIV-A(enfermedad de Morquio) es una enfermedad autosómica recesiva por acúmulo lisosomal, causada por mutaciones en el gen de la $\mathrm{N}$-acetylgalactosamine-6-sulfato sulfatasa, que resulta en la falta de catabolismo de dos glicosaminoglicanos, el queratán y condroitín sulfato. Las manifestaciones clínicas varían desde un fenotipo grave a una forma atenuada. El acúmulo de sustrato se expresa como displasia ósea, baja estatura, inestabilidad atlantoaxoidea y compresión cervical. El compromiso cardíaco, respiratorio, auditivo y ocular también ha sido descrito como parte de la enfermedad. La elosulfasa alfa es el único tratamiento específico para la enfermedad de Morquio. Los estudios recientemente publicados muestran que la elosulfasa alfa en niños y adultos logra mejorar la resistencia física y reducir en forma sostenida los niveles de queratán sulfato. Los datos del registro Morquio internacional mostrarán la evolución natural de los pacientes, así como también la eficacia a largo plazo de la elosulfasa alfa. Palabras clave: elosulfasa alfa, enfermedad de Morquio, mucopolisacaridosis $I V-A$, terapia de reemplazo enzimático.
\end{abstract}

\section{ABSTRACT}

Mucopolysaccharidosis type IV-A (Morquio A disease) is an autosomal recessive lysosomal storage disease caused by mutations in the gene encoding the $\mathrm{N}$-acetylgalactosamine-6-sulfate sulfatase, that results in impaired catabolism of two glycosaminoglycans, chondroitin-6-sulfate and keratan sulfate. Clinical presentations reflecta spectrum of progression from a severe phenotype to an attenuated expression. Accumulation of substrate manifests predominantly as short stature and skeletal dysplasia, including atlantoaxial instability and cervical cord compression. Other abnormalities in the visual, auditory, cardiovascular and respiratory systems can also affect individuals with Morquio disease. Elosulfase alfa showed in clinical trials in children and adults a significant and sustained improvement in endurance and urinary levels of keratan sulfate. Data from the ongoing observational, multinational Morquio A Registry Study will provide valuable information on the long-term efficacy and safety of elosulfase alfa in patients, as well as on the natural history of this very rare disease.

Key words: elosulfasa alfa, Morquio disease, mucopolysaccharidosis $I V-A$, enzyme replacement therapy.

http://dx.doi.org/10.5546/aap.2015.359

\section{INTRODUCCIÓN}

La mucopolisacaridosis tipo IV-A (MPS IV-A), también conocida como enfermedad de Morquio, es una enfermedad por depósito lisosomal con herencia autosómica recesiva. ${ }^{1}$ Fue descrita en 1929 en forma separada por Luis Morquio (pediatra de Uruguay) y James Brailsford (radiólogo de Inglaterra). Su incidencia en la población general es de $1 / 200000$, aunque se han reportado desde 1/76000 nacidos vivos en el norte de Irlanda hasta 1/640000 en el oeste de Australia. ${ }^{2}$ La MPS IV-A es el resultado de mutaciones en el gen que codifica para la enzima lisosomal $\mathrm{N}$-acetylgalactosamina6-sulfato sulfatasa (GALNS), lo que deriva en una reducción o ausencia del catabolismo de dos glicosaminoglicanos: el condroitín sulfato y el queratán sulfato. ${ }^{3}$

Como en las otras MPS, existen distintas formas de presentación, que varían desde una forma grave (también llamada clásica) hasta una forma leve (o llamada atenuada). ${ }^{1}$ La primera variable que distingue a estos fenotipos es la presencia de manifestaciones típicas antes del año 
de vida en la forma grave y una aparición de síntomas recién en la segunda década de vida en la forma atenuada. Otras diferencias serán la estatura ( $\leq 120 \mathrm{~cm}$ en la forma grave $\mathrm{y} \geq 140 \mathrm{~cm}$ en la forma atenuada), el grado de compromiso orgánico y la cuantificación del sustrato, entre las más características. ${ }^{3}$

En 1976, se describió un caso compatible con enfermedad de Morquio, pero la enzima deficiente era la beta-galactosidasa ácida. ${ }^{4}$ Esta situación fue corroborada por otro autor, ${ }^{5}$ por lo que hoy se define la MPS IV en tipo A (deficiencia de GALNS) y tipo B (deficiencia de betagalactosidasa). ${ }^{6}$ Con la aprobación por parte de la Administración de Medicamentos y Alimentos (Food and Drug Administration; FDA, por sus siglas en inglés) al inicio de 2014 de la terapia específica para la MPS IV-A, esta entidad ha generado un mayor interés en la comunidad médica.

\section{OBJETIVO}

Realizar una revisión de las manifestaciones clínicas, el diagnóstico y las novedades del tratamiento de la MPS IV-A.

\section{MANIFESTACIONES CLÍNICAS}

Debido a que el queratán sulfato y el condroitín sulfato son el mayor componente de proteoglicanos en cartílagos y huesos, la manifestación más evidente de la MPS IV-A será la displasia esquelética, definida también como disostosis múltiple (DM). ${ }^{2}$

Los síntomas de inicio característicos que se reportan con mayor frecuencia incluyen deformidad ósea, baja talla y alteraciones de la marcha. La edad de inicio en más del $50 \%$ de los pacientes con formas graves es de 2,2 años, aunque el diagnóstico suele retrasarse por, al menos, 3 años, lo que lleva a una media de edad para el diagnóstico de 4,9 años. ${ }^{7}$ Otros signos y síntomas, como cifosis y pectus carinatum, suelen estar presentes aun antes del año de vida, aunque su presencia no suele ser suficiente como para llegar al diagnóstico en la mayoría de los casos. ${ }^{7}$ Luego del primer año de vida, la deformidad espinal (giba) y el trastorno en la marcha son los signos más tangibles.

Los pacientes con fenotipo grave presentan una velocidad de crecimiento reducida, que comienza a manifestarse luego de los 24 meses y suele detenerse a los 7 u 8 años. ${ }^{8}$

El registro Morquio A internacional ha podido colectar datos clínicos que agregaron al conocimiento de la enfermedad, su progresión e historia natural. Hasta diciembre de 2011, se contaba con información de 399 casos. El análisis comparativo de la talla de 190 mujeres y 209 varones con MPS IV-A con un grupo control sano mostró que, al nacer, la talla media fue de $52 \mathrm{~cm}$ en varones y de $51,9 \mathrm{~cm}$ en mujeres, lo que corresponde a un $+0,75 \mathrm{DE} \mathrm{y}+0,98 \mathrm{DE}$, respectivamente. A los dos años, la talla media se mantuvo dentro de los valores normales, ya que luego la talla cae a $-2 \mathrm{DE}$ en ambos géneros. La estatura media a los 18 años fue de 122,4 cm en hombres $( \pm 21,5 \mathrm{~cm})$ y de $113,1 \mathrm{~cm}$ en mujeres $( \pm 22,6 \mathrm{~cm})$. El peso medio al nacer para varones y niñas fue de 3,5 y de $3,4 \mathrm{~kg}$, respectivamente. A la edad de 18 años, fue de 40,7 en hombres y de $36,7 \mathrm{~kg}$ en mujeres, mientras que el grupo control reportó $67,2 \mathrm{~kg}$ y 56,2 kg en hombres y mujeres, respectivamente. La diferencia mayor a $-2 \mathrm{DE}$ fue evidente desde los 12 años de edad para ambos géneros respecto al peso de los pacientes. ${ }^{7}$

La enfermedad de Morquio, como la MPS tipo VI, son descritas como las únicas MPS sin compromiso cognitivo. ${ }^{9,10}$ Cabe destacar que, en algunos casos en los que por compresión bulbomedular se desarrolla hidrocefalia, pueden ser evidentes signos de deterioro intelectual. Recientemente, un estudio ha mostrado valores por debajo de la media esperada en tests neurocognitivos en algunos niños con MPS IV-A. ${ }^{11}$

Una característica compartida entre todas las MPS es la presencia de DM. Si bien hay diferencias en la presentación clínica, se han descrito radiológicamente los siguientes hallazgos: silla turca en forma de J, aumento del espacio diploico en la calota, clavículas cortas y gruesas, cuerpo vertebral en pico y borde posterior festoneado (Figura 1), alas ilíacas redondeadas, coxa valga (Figura 2), epífisis

FIGURA 1. Hipoplasia de cuerpos vertebrales y giba lumbar característica

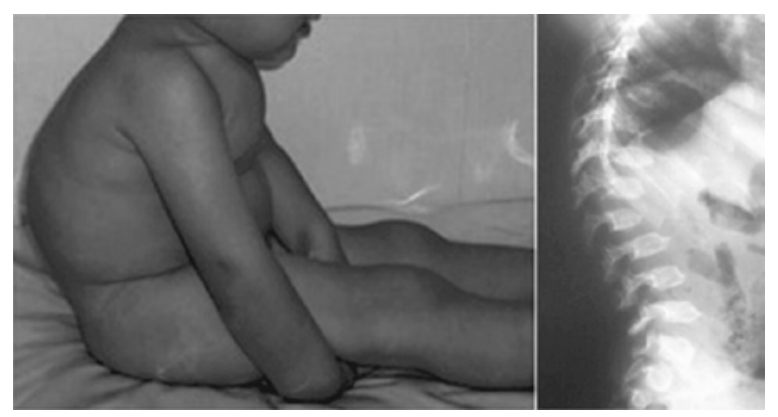


hipoplásicas (predominantemente, a nivel distal de cúbito y radio) (Figura 3), engrosamiento y acortamiento diafisario, retraso de la edad ósea con respecto a la edad cronológica en los huesos de la mano (disociación carpo-epifisaria) y osificación tardía o no presente del escafoides (Figura 4)..$^{1-3}$

El análisis de la frecuencia de los distintos componentes de la DM sobre la base del registro Morquio muestra en orden decreciente genu valgo, giba lumbar (Figura 1), pectumcarinatum, deformidades en las muñecas, la cadera, el fémur y el tobillo.?

FIGURA 2. Coxa valga asociada a hipoplasia de la cabeza del fémur

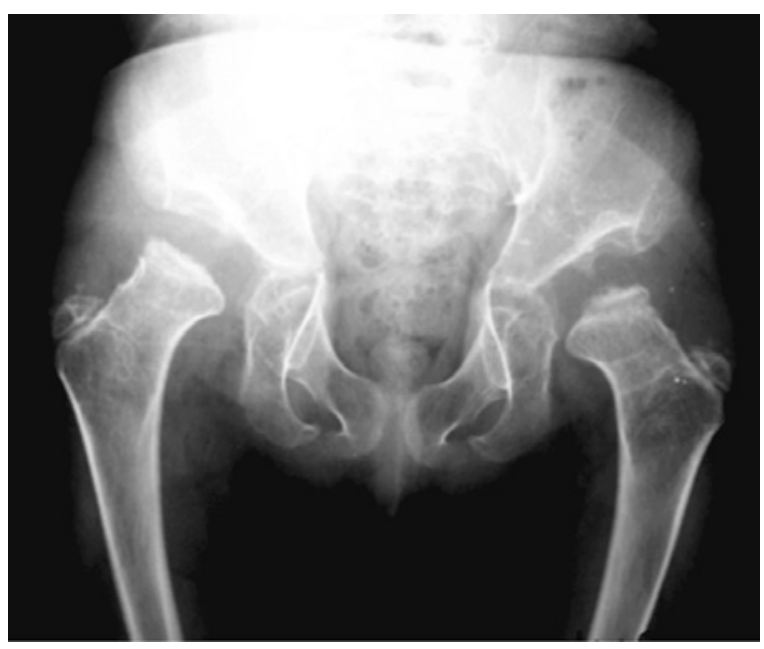

FIGURA 3. Hipoplasia epifisaria en el cúbito y el radio

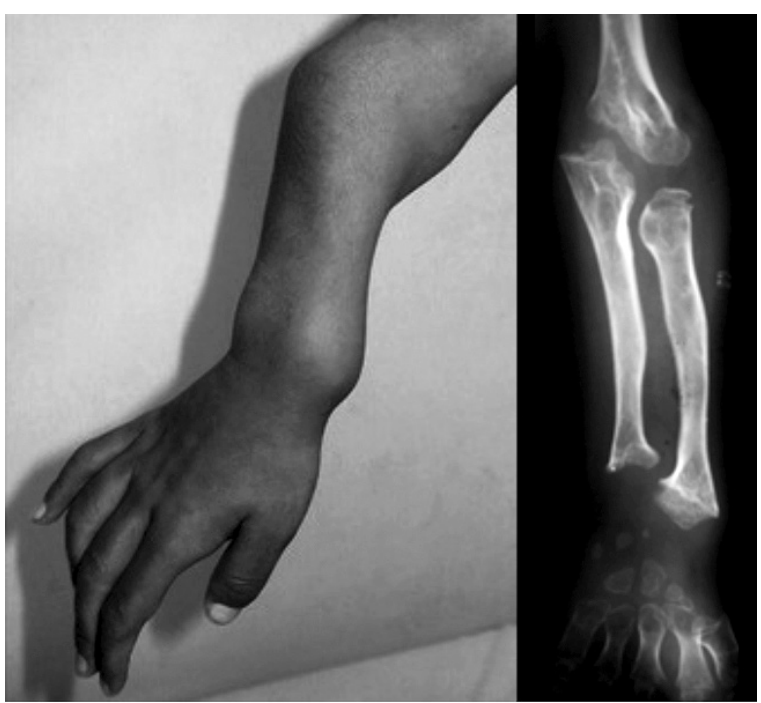

Posiblemente, otra manifestación característica y que solo se evidencia en la MPS IV-A es la hiperlaxitud articular (Figura 4). El resto de las MPS muestran como patrón característico la retracción articular, que configura la típica mano en "garra", hallazgo que debe permanecer ausente en la MPS IV-A.

La combinación de hipoplasia vertebral, platispondilia y cifosis asociada a la hiperlaxitud y engrosamiento ligamentario por depósito de glicosaminoglicanos son los principales mecanismos fisiopatológicos del compromiso espinal en dos niveles, cervical y dorso-lumbar. ${ }^{12}$ A nivel medial, la unión atlanto-axoidea está constituida por la apófisis odontoidea y el anillo formado por el arco anterior del atlas y el ligamento transverso. Esta unión permite el $50 \%$ de la rotación cervical lateral. Un ligamento transverso indemne asociado a los ligamentos alares laterales mantiene la integridad de la articulación y limita el movimiento posterior de la apófisis odontoidea, lo que podría comprimir la médula espinal en ese nivel. Sin embargo, la hiperlaxitud ligamentaria asociada a la hipoplasia de la apófisis odontoidea de la MPS IV-A lleva, muchas veces, a un movimiento independiente entre el arco anterior del atlas y odontoides, lo que se define como inestabilidad articular. ${ }^{13}$ Durante la flexión, el arco anterior del atlas se dirige a un plano anteroinferior, por lo que un ligamento laxo e incontinente no puede llevar la apófisis odontoidea hacia el mismo plano y se genera un pinzamiento por parte del arco posterior de atlas sobre la cara dorsal de la médula cervical. En casos de compromiso grave

FIgURA 4. A. Hiperlaxitud articular en la muñeca; B. Retraso de la edad ósea en los huesos de la mano (disociación carpo-epifisaria) y osificación no presente del escafoides

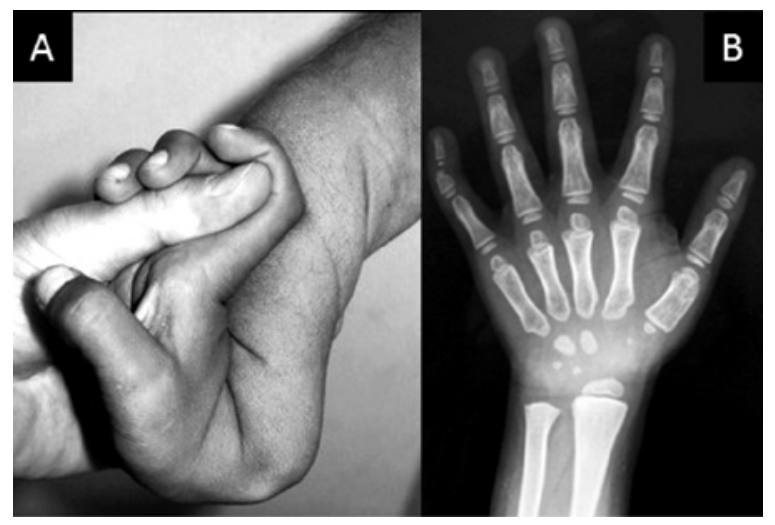


de esta articulación, se producirá en los niños un aumento mayor de $2,5 \mathrm{~mm}$ y en los adultos de $5 \mathrm{~mm}$ del intervalo atlanto-odontoideo, lo que se conoce como subluxación horizontal atlantoaxoidea. El resultado de esta complicación es la compresión espinal, que puede derivar en cuadriparesia o cuadriplejía (Figura 5).

La protrusión de los discos intervertebrales es otro mecanismo que, asociado a los depósitos de glicosaminoglicanos en el ligamento longitudinal posterior y amarillo e hipoplasia ósea, produce estenosis del canal espinal, particularmente en la región dorso-lumbar (D10-L1), en la que el grosor de la médula espinal es mayor. ${ }^{12}$ Este sector es más propenso a cifosis debido a una transición de un área más rígida (caja torácica) a otra más flexible (Figura 1).

Las cirugías más frecuentemente reportadas de un total de 326 pacientes evaluados están relacionadas con la cabeza y el cuello. La más frecuente es la descompresión y estabilización de la columna cervical. Las cirugías más tempranas fueron la adenoidectomía y amigdalectomía para el manejo de la obstrucción ventilatoria. A continuación de estos procedimientos, se describen osteotomía femoral, reemplazo de cadera y tubos de ventilación en el oído (diábolo). ${ }^{7}$

El compromiso respiratorio es de causa multifactorial y se atribuye a la obstrucción de la vía respiratoria alta y baja, a la restricción de la caja torácica y a la mielopatía cervical. La deformidad de la caja torácica como resultado del pectum carinatum y la cifoescoliosis limita la expansión pulmonar y el desplazamiento del diafragma hacia el tórax por la baja estatura, y la hepatomegalia y/o esplenomegalia agregan más al componente restrictivo. ${ }^{3}$ El depósito de glicosaminoglicanos en las amígdalas y las paredes de la vía aérea superior predispone a los

FIGURA 5. A. Reconstrucción tridimensional de columna cervical con hipoplasia de apófisis odontoidea; B. Mielopatía compresiva con mielomalacia a nivel C1-C2

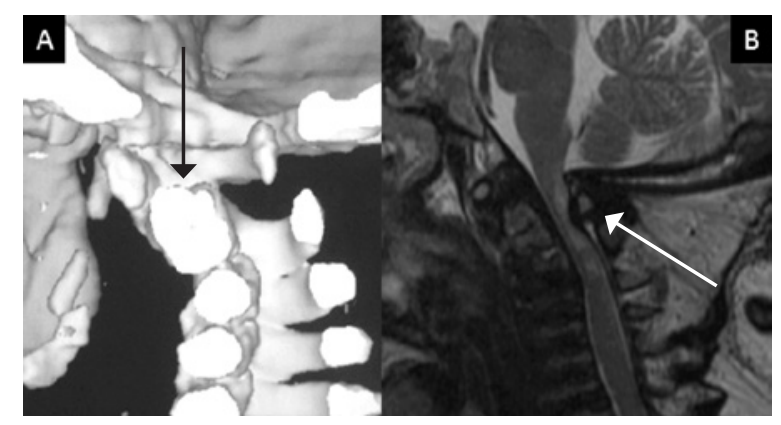

pacientes a las infecciones respiratorias repetidas (otitis y sinusitis). Por último, el síndrome de apnea obstructiva nocturna suele estar presente y no ser evidente en etapas tempranas, por lo que se sugiere la evaluación con polisomnografía anual. ${ }^{14}$

La manifestación más frecuente relacionada con la visión es la opacidad corneal, que es menos progresiva que en otras MPS. El depósito de glicosaminoglicanos en la córnea se define como un patrón fino granular que se observa en luz de hendidura y, en casos muy graves, puede ser visto en forma oblicua con linterna. ${ }^{15}$ El glaucoma tanto de ángulo abierto como estrecho y las cataratas han sido descritos, pero no requieren cirugía correctiva en su gran mayoría. ${ }^{16}$

La pérdida auditiva se describe con frecuencia y suele ser el resultado de las repetidas infecciones respiratorias de la vía aérea superior, deformidades de los huesecillos del oído medio y del compromiso neurosensitivo por acúmulo de glicosaminoglicanos en estructuras de la cóclea y del octavo par craneal. ${ }^{3}$ La necesidad de audífonos ha sido reportada desde la adolescencia en la mayoría de los casos. ${ }^{17}$

Si bien el compromiso digestivo de la MPS IV-A está dado por la presencia de hernias umbilical e inguinal y hepatomegalia, estas manifestaciones suelen ser menos frecuentes y serias en comparación con las otras MPS. ${ }^{18}$

Una complicación característica y bien descrita en MPS IV-A es la malformación dentaria. El defecto estructural del esmalte dental ha sido demostrado solo en MPS IV-A, en la que el depósito de queratán sulfato y condroitín sulfato en lisosomas de ameloblastos en fase secretoria serían razones suficientes para explicar la mayor incidencia de caries. ${ }^{19,20}$

El compromiso cardiovascular suele ser menos descrito en la MPS IV-A en comparación con las MPS I, II y VI. Algunas series reportan hasta un $40 \%$ de casos con engrosamiento de las válvulas mitral y aórtica en una población con una media de edad de 12,5 años. ${ }^{21}$ Otros estudios que no diferencian entre MPS IV-A y B mostraron estenosis valvular entre el $7 \mathrm{y}$ el $9 \%$ e insuficiencia valvular entre el $17 \mathrm{y}$ el $26 \%$ de los casos estudiados. ${ }^{22}$

A la fecha, se ha reportado una mujer embarazada con fenotipo grave de MPS IV-A, quien concluyó su embarazo en la semana 28 por disnea e intolerancia al dolor. La hija evolucionó con enfermedad de membrana hialina, anemia, hiperbilirrubinemia, apneas y displasia broncopulmonar. Luego del tratamiento de estas complicaciones, la evolución fue favorable. ${ }^{23}$ 


\section{DIAGNÓSTICO}

El diagnóstico de MPS IV-A requiere demostrar la disminución de la actividad de la GALNS en leucocitos o fibroblastos. Ante la dificultad que puede presentarse para el traslado de este tipo de material, existe la posibilidad de utilizar gotas de sangre seca en papel de filtro para su análisis. Este método es considerado de pesquisa y puede ocasionar resultados falsos positivos. ${ }^{24}$

La actividad de la GALNS también puede estar disminuida en otra patología: el déficit de múltiples sulfatasas. Para poder demostrar que la deficiencia de GALNS se debe a la enfermedad de Morquio A, se debe realizar en forma concomitante el análisis de otra sulfatasa (arilsulfatasa A o iduronato-2-sulfatasa). ${ }^{24}$

En forma simultánea, se debe realizar el estudio de los glicosaminoglicanos en la orina. El análisis cuantitativo mide la cantidad total de mucopolisacáridos excretados, teniendo presente que existen pacientes con MPS que pueden excretar cantidades normales de estos metabolitos. En el análisis cualitativo de los glicosaminoglicanos en la orina mediante cromatografía, se evalúa el perfil de mucopolisacáridos excretados. Este estudio brinda información valiosa para orientar el diagnóstico y puede identificar la presencia de queratán sulfato aun en pacientes con cantidades normales de glicosaminoglicanos en la orina.

El test molecular (genético) no es obligatorio para el diagnóstico definitivo en la mayoría de los casos y es de utilidad para el consejo genético actualmente. Distintos polimorfismos han sido descritos a la fecha, por lo que, ante la presencia de una mutación no descrita, es necesario el uso de herramientas predictivas para definir la patogenicidad de la mutación. ${ }^{25}$

\section{TRATAMIENTO}

Antes de la aprobación de la terapia de reemplazo enzimático (TRE), se consideraba el tratamiento de la MPS IV-A como paliativo. El trasplante de médula ósea no ha demostrado evitar la aparición de las manifestaciones esqueléticas y, dada la alta morbimortalidad del procedimiento, no se sugiere realizar de rutina este tratamiento. ${ }^{26}$

El 14 de febrero de 2014, la FDA aprobó la primera terapia recombinante para MPS IV-A (elosulfasa alfa-Vimizym ${ }^{\circledR}-$ ), y, el 20 de febrero, la Agencia de Medicina Europea recomendó su aprobación para la misma indicación. ${ }^{27} \mathrm{La}$ elosulfasa alfa tiene adherida una cadena de oligosacárido terminal (manosa-6-fosfato), por la cual es reconocida e internalizada por las células luego de su infusión intravenosa. Una vez internalizada, la actividad enzimática específica se incrementa en forma dosis-dependiente y se logra el catabolismo del queratán y condroitín sulfato. ${ }^{28}$ Antes de los ensayos que llevaron a la aprobación final del tratamiento, este mecanismo de acción fue probado en condrocitos aislados de pacientes con MPS IV-A, en los que el nivel endógeno de GALNS era indetectable y el nivel de queratán sulfato era 11 veces mayor que el normal. Luego del tratamiento con elosulfasa alfa, los niveles enzimáticos fueron normales, el depósito de queratán sulfato disminuyó y hubo una modulación positiva de genes condrogénicos con un incremento en la expresión de colágeno II, colágeno $\mathrm{X}$, agrecano y SOX9. ${ }^{28}$

La eficacia de la elosulfasa alfa fue evaluada en un ensayo aleatorizado, doble-ciego, controlado con placebo y multinacional. ${ }^{28}$ Este estudio incluyó un total de 176 pacientes, todos mayores de 5 años de edad, con una media de 11,9 años. Como criterio de inclusión, los pacientes debían poder caminar $\geq 30$ metros $\mathrm{y} \leq 325$ metros en el test de 6 minutos ( 6 minutes walking test; 6MWT, por sus siglas en inglés). Los pacientes fueron aleatorizados en 3 ramas: elosulfasa alfa de 2 $\mathrm{mg} / \mathrm{kg}$ por semana (grupo A), elosulfasa alfa de $2 \mathrm{mg} / \mathrm{kg}$ cada 2 semanas (grupo B) y placebo. El punto de eficacia terminal fue la diferencia en el 6MWT a la semana 24 de tratamiento. Los puntos de eficacia secundarios fueron los niveles de queratán sulfato en la orina y los escalones subidos por escalera en 3 minutos (3 Minute Stair Climb Test; 3MSCT, por sus siglas en inglés). Los puntos de eficacia terciarios fueron los parámetros funcionales respiratorios, un puntaje compuesto de 6MWT/3MSCT/ventilación voluntaria máxima, evaluaciones antropométricas y escalas de calidad de vida. La edad media del grupo A fue de 13,1 años; del grupo B, de 15,3 años; y del grupo placebo, de 15 años; y la distancia media recorrida basal fue de 204 metros, 206 metros y 212 metros, respectivamente. Al final del estudio, la diferencia del punto de eficacia primario fue logrado por el grupo A, con un aumento significativo desde la evaluación basal a la semana 24 en comparación con el grupo placebo (37 vs. 14 metros; $p=0,0174$ ). El grupo B no logró una diferencia significativa con el grupo placebo. El 3MSCT no mostró diferencias significativas entre los 3 grupos. Los niveles de 
queratán sulfato urinarios disminuyeron un $40,7 \%$ en el grupo A y un $30,2 \%$ en el grupo B con respecto al grupo placebo, utilizando el algoritmo de mínimos cuadrados (least mean square; LMS, por sus siglas en inglés), lo que se considera significativo. Finalmente, 8 de 10 puntos terciarios fueron favorables para el grupo A con respecto al grupo placebo. ${ }^{27,28}$

Los efectos adversos descritos con mayor frecuencia fueron escalofríos, cefaleas, náuseas y fatiga. Ningún paciente debió ser retirado de la fase 3 y los pocos eventos adversos serios reportados fueron relacionados con las complicaciones conocidas de la MPS IV-A. Raramente se han reportado reacciones inmunológicas graves.

\section{REFERENCIAS}

1. Hendriksz CJ, Lavery C, Coker M, Ucar SK, et al. Burden of disease in patients with Morquio A syndrome: results from an international patient-reported outcomes survey. Orphanet J Rare Dis 2014;9:32.

2. Leadley RM, Lang S, Misso K, Bekkering T, et al. A systematic review of the prevalence of Morquio A syndrome: challenges for study reporting in rare diseases. Orphanet J Rare Dis 2014;9(1):173.

3. Hendriksz CJ, Al-Jawad M, Berger KI, Hawley SM, et al. Clinical overview and treatment options for non-skeletal manifestations of mucopolysaccharidosis type IVA. J Inherit Metab Dis 2013;36(2):309-22.

4. O'Brien JS, Gugler E, Giedion A, Wiessmann U, et al. Spondyloepiphyseal dysplasia, corneal clouding, normal intelligence and acid beta-galactosidase deficiency. Clin Genet 1976;9(5):495-504.

5. Arbisser AI, Donnelly KA, Scott CI Jr, DiFerrante N, et al. Morquio-likesyndrome with beta galactosidase deficiency and normal hexosamine sulfatase activity: mucopolysacchariodosis IVB. Am J Med Genet 1977;1(2):195-205.

6. Di Cesare A, Di Cagno A, Moffa S, Teresa P, et al. A description of skeletal manifestation in adult case of morquio syndrome: radiographic and MRI appearance. Case Rep Med 2012;2012:324596.

7. Montaño AM, Tomatsu S, Gottesman GS, Smith M, et al. International Morquio A Registry: clinical manifestation and natural course of Morquio A disease. J Inherit Metab Dis 2007;30(2):165-74.

8. Montaño AM, Tomatsu S, Brusius A, Smith M, etal. Growth charts for patients affected with Morquio A disease. Am J Med Genet A 2008;146A(10):1286-95.

9. Lachman RS, Burton BK, Clarke LA, Hoffinger S, et al. Mucopolysaccharidosis IVA (Morquio A syndrome) and VI (Maroteaux-Lamy syndrome): under-recognized and challenging to diagnose. Skeletal Radiol 2014;43(3):359-69.

10. Hendriksz CJ, Harmatz P, Beck M, Jones S, et al. Review of clinical presentation and diagnosis of mucopolysaccharidosis IVA. Mol Genet Metab 2013;110(12):54-64.
11. Davison JE, Kearney S, Horton J, Foster K, et al. Intellectual and neurological functioning in Morquio syndrome (MPS IVa). J Inherit Metab Dis 2013;36(2):323-8.

12. SolankiGA, Martin KW, Theroux MC, LampeC, etal.Spinal involvement in mucopolysaccharidosis IVA (MorquioBrailsford or Morquio A syndrome): presentation, diagnosis and management. J Inherit Metab Dis 2013;36(2):339-55.

13. Charrow J, Alden TD, Breathnach CA, Frawley GP, et al. Diagnostic evaluation, monitoring, and perioperative management of spinal cord compression in patients with Morquio syndrome. Mol Genet Metab 2015;114(1):11-8.

14. Pelley CJ, Kwo J, Hess DR. Tracheomalacia in an adult with respiratory failure and Morquio syndrome. Respir Care 2007;52(3):278-82.

15. CouprieJ,Denis P, GuffonN, Reynes N, etal. Manifestations ophtalmologiques de la maladie de Morquio. JFr Ophtalmol 2010;33(9):617-22.

16. Leslie T, Siddiqui MA, Aitken DA, Kirkness CM, et al. Morquio syndrome: electron microscopic findings. $\mathrm{Br} \mathrm{J}$ Ophthalmol 2005;89(7):925-6.

17. Tunkel D, Alade Y, Kerbavaz R, Smith B, et al. Hearing loss in skeletal dysplasia patients. Am J Med Genet A 2012;158A(7):1551-5.

18. Tomatsu S, Montaño AM, Oikawa H, Smith $M$, et al. Mucopolysaccharidosis type IVA (Morquio A disease): clinical review and current treatment. Curr Pharm Biotechnol 2011;12(6):931-45.

19. Yamakoshi $Y, \mathrm{Hu}$ JC, Liu S, Sun X, et al. Porcine $\mathrm{N}$-acetylgalactosamine 6-sulfatase (GALNS) cDNA sequence and expression in developing teeth. Connect Tissue Res 2002;43(2-3):167-75.

20. Kuratani T, Miyawaki S, Murakami T, Takano-Yamamoto T. Early orthodontic treatment and long-term observation in a patient with Morquio syndrome. Angle Orthod 2005;75(5):881-7.

21. John RM, Hunter D, Swanton RH. Echocardiographic abnormalities in type IV mucopolysaccharidosis. Arch Dis Child 1990;65(7):746-9.

22. LealGN, dePaula AC, LeoneC, Kim CA.Echocardiographic study of paediatric patients with mucopolysaccharidosis. Cardiol Young 2010;20(3):254-61.

23. Salinas H, Preisler J, Astudillo J, Cerda S, et al. Síndrome de Morquio (mucopolisacaridosis tipo IV) y embarazo. Rev Chil Obstet Ginecol 2005;70(6):400-3.

24. Wood TC, Harvey K, Beck M, Burin MG, et al. Diagnosing mucopolysaccharidosis IVA. J Inherit Metab Dis 2013;36(2):293-307.

25. Tomatsu S, Montaño AM, Nishioka T, Gutierrez MA, et al. Mutation and polymorphism spectrum of the GALNS gene in mucopolysaccharidosis IVA (Morquio A). Hum Mutat 2005;26(6):500-12.

26. Tomatsu S, Yasuda E, Patel P, Ruhnke K, et al. Morquio A syndrome: diagnosis and current and future therapies. Pediatr Endocrinol Rev 2014;12(Suppl 1):141-51.

27. Sanford M, Lo JH. Elosulfase alfa: first global approval. Drugs 2014;74(6):713-8.

28. Lyseng-Williamson KA. Elosulfase Alfa: a review of its use in patients with mucopolysaccharidosis type IVA(Morquio A syndrome). BioDrugs 2014;28(5):465-75. 